\title{
Comparison of Macintosh Laryngoscopy in Bed-up-Head- Elevated Position With GlideScope Laryngoscopy: A Randomized, Controlled, Noninferiority Trial
}

\author{
Samuel E. H. Tsan, MD, BMedSc, ${ }^{*}+$ Siu M. Lim, MBBS, M(Anaes), ${ }^{*}$ \\ Mohd F. Z. Abidin, MD, M(Anaes), * Shahmini Ganesh, MD,* and Chew Y. Wang, MBChB, FRCA*
}

See Article, p 208

\begin{abstract}
BACKGROUND: Approximately half of all difficult tracheal intubations (DTIs) are unanticipated; hence, proper positioning during intubation is critical to increase the likelihood of success. The bed-up-head-elevated (BUHE) intubation position has been shown to improve laryngeal view, reduce airway complications, and prolong safe apneic time during intubation. In this study, we sought to determine whether the BUHE intubation position is noninferior to Glidescope (GLSC)assisted intubation with regard to laryngeal exposure.

METHODS: A total of 138 American Society of Anesthesiologists (ASA) I to III patients were randomly assigned into 2 groups and underwent baseline laryngoscopy in the sniffing position. Group BUHE patients $(n=69)$ were then intubated in the BUHE position, while group GLSC patients $(n=69)$ were intubated using GLSC laryngoscopy. Laryngeal exposure was measured using Percentage of Glottic Opening (POGO) score and Cormack-Lehane (CL) grading, and noninferiority will be declared if the difference in mean POGO scores between both groups do not exceed $-15 \%$ at the lower limit of a $98 \%$ confidence interval $(\mathrm{Cl})$. Secondary outcomes measured included time required for intubation (TRI), number of intubation attempts, use of airway adjuncts, effort during laryngoscopy, and complications during intubation.

RESULTS: Mean POGO score in group BUHE was $80.14 \% \pm 22.03 \%$, while in group GLSC it was $86.45 \% \pm 18.83 \%$, with a mean difference of $-6.3 \%(98 \% \mathrm{Cl},-13.2 \%$ to $0.6 \%)$. In both groups, there was a significant improvement in mean POGO scores when compared to baseline laryngoscopy in the sniffing position (group BUHE, 25.8\% $\pm 4.7 \%$; group GLSC, $30.7 \% \pm 6.8 \%$ ) $(P<.0001)$. The mean TRI was $36.23 \pm 14.41$ seconds in group BUHE, while group GLSC had a mean TRI of $44.33 \pm 11.53$ seconds $(P<.0001)$. In patients with baseline CL 3 grading, there was no significant difference between mean POGO scores in both groups (group BUHE, $49.2 \%$ $\pm 19.6 \%$ versus group GLSC, $70.5 \% \pm 29.7 \% ; P=.054)$.

CONCLUSIONS: In the general population, BUHE intubation position provides a noninferior laryngeal view to GLSC intubation. The laryngeal views obtained in both approaches were superior to the laryngeal view obtained in the sniffing position. In view of the many advantages of the BUHE position for intubation, the lack of proven adverse effects, the simplicity, and the costeffectiveness, we propose that clinicians should consider the BUHE position as the standard intubation position for the general population. (Anesth Analg 2020;131:210-9)
\end{abstract}

\section{KEY POINTS}

Question: Does the bed-up-head-elevated (BUHE) position provide a noninferior laryngeal view to Glidescope (GLSC) laryngoscopy?

Findings: The BUHE position is able to provide a noninferior laryngeal exposure when compared with GLSC laryngoscopy, within the bounds of the margin of noninferiority set at $-15 \%$. Meaning: The BUHE position can be a standard position for intubation because it provides a laryngeal view that is not inferior to the laryngeal view obtained by video-assisted laryngoscopy (VAL).

\section{GLOSSARY}

ASA = American Society of Anesthesiologists; $\mathbf{B M I}=$ body mass index; $\mathbf{B U H E}=$ bed-up-headelevated; $\mathbf{C I}=$ confidence interval; $\mathbf{C L}=$ Cormack-Lehane; CONSORT = Consolidated Standards of Reporting Trials; $\mathbf{D T I}=$ difficult tracheal intubations; ETI = endotracheal intubation; $\mathbf{G A}=$ general anesthesia; GLSC = Glidescope; IDS = Intubation Difficulty Scale; IV = intravenous; $\mathbf{M A P}=$ mean arterial pressure; POGO = Percentage of Glottic Opening; SD = standard deviation; $\mathbf{S p o}_{\mathbf{2}}=$ oxygen saturation; SSP = supine and sniffing position; TMD = thyromental distance; TRI = time required for intubation; VAL = video-assisted laryngoscopy

From the *Department of Anaesthesiology, Faculty of Medicine, University of Malaya, Kuala Lumpur, Malaysia; and +Department of Anaesthesiology, Faculty of Medicine and Health Sciences, University of Malaysia Sarawak, Sarawak, Malaysia.

Accepted for publication June 24, 2019.

Funding: This study was funded by the Malaysian Society of Anaesthesiologists K. Inbasegaran fund.

Copyright (C) 2019 International Anesthesia Research Society DOI: 10.1213 /ANE.0000000000004349
The authors declare no conflicts of interest.

Clinical trial registry: ClinicalTrials.gov (Registration identifier: NCT03357679).

Reprints will not be available from the authors.

Address correspondence to Samuel E. H. Tsan, MD, BMedSc, Department of Anaesthesiology, Faculty of Medicine and Health Sciences, University of Malaysia Sarawak, Jalan Datuk Mohammad Musa, 94300 Kota Samarahan, Sarawak, Malaysia. Address e-mail to tehsamuel@unimas.my. 